\title{
Design and Synthesis of Target-Responsive Hydrogel for Portable Visual Quantitative Detection of Uranium with a Microfluidic Distance-Based Readout Device
}

\author{
Yishun Huang ${ }^{a}$, Luting Fang ${ }^{a}$, Zhi Zhu ${ }^{a}$, Yanli Ma ${ }^{a}$, Leiji Zhou ${ }^{a}$, Xi Chen ${ }^{b}$, \\ Dunming $X u^{b}$, Chaoyong Yang ${ }^{a} *$
}

a: MOE Key Laboratory of Spectrochemical Analysis \& Instrumentation, Collaborative Innovation Center of Chemistry for Energy Materials, Key Laboratory for Chemical Biology of Fujian Province, State Key Laboratory of Physical Chemistry of Solid Surfaces, College of Chemistry and Chemical Engineering, Xiamen University Xiamen 361005 (China)

b: Xiamen Entry-Exit Inspection and Quarantine Bureau, Xiamen 361026, China

RECEIVED DATE (to be automatically inserted after your manuscript is accepted if required according to the journal that you are submitting your paper to)

* To whom correspondence should be addressed. Tel: (+86) 592-218-7601, E-mail: cyyang@xmu.edu.cn 


\section{Abstract}

Due to uranium's increasing exploitation in nuclear energy and its toxicity to human health, it is of great significance to detect uranium contamination. In particular, development of a rapid, sensitive and portable method is important for personal health care for those who frequently come into contact with uranium ore mining or who investigate leaks at nuclear power plants. The most stable form of uranium in water is uranyl ion $\left(\mathrm{UO}_{2}{ }^{2+}\right)$. In this work, a $\mathrm{UO}_{2}{ }^{2+}$ responsive smart hydrogel was designed and synthesized for rapid, portable, sensitive detection of $\mathrm{UO}_{2}{ }^{2+}$. $\mathrm{A} \mathrm{UO}_{2}{ }^{2+}$ dependent DNAzyme complex composed of substrate strand and enzyme strand was utilized to crosslink DNA-grafted polyacrylamide chains to form a DNA hydrogel. Colorimetric analysis was achieved by encapsulating gold nanoparticles (AuNPs) in the DNAzyme-crosslinked hydrogel to indicate the concentration of $\mathrm{UO}_{2}{ }^{2+}$. Without $\mathrm{UO}_{2}{ }^{2+}$, the enzyme strand is not active. The presence of $\mathrm{UO}_{2}{ }^{2+}$ in the sample activates the enzyme strand and triggers the cleavage of the substrate strand from the enzyme strand, thereby decreasing the density of crosslinkers and destabilizing the hydrogel, which then releases the encapsulated AuNPs. As low as $100 \mathrm{nM} \mathrm{UO}_{2}{ }^{2+}$ was visually detected by the naked eye. The target-responsive hydrogel was also demonstrated to be applicable in natural water spiked with $\mathrm{UO}_{2}{ }^{2+}$. Furthermore, to avoid the visual errors caused by naked eye observation, a previously developed volumetric bar-chart chip (V-Chip) was used to quantitatively detect $\mathrm{UO}_{2}{ }^{2+}$ concentrations in water by encapsulating Au-Pt nanoparticles in the hydrogel. The $\mathrm{UO}_{2}{ }^{2+}$ concentrations were visually quantified from the travelling distance of ink-bar on the V-Chip. The method can be used for portable and quantitative detection of uranium in field applications without skilled operators and sophisticated instruments.

Keywords: Uranium, DNA hydrogel, portable detection, DNAzyme. 


\section{Introduction}

Uranium is a ubiquitous trace radioactive element that is used as the main fuel in nuclear reactors. With its growth in the nuclear power industry and in nuclear weapons (Domingo 2001a), the worldwide uranium consumption steadily increased. Meanwhile, uranium pollution induced by the mining of uranium ores, the inappropriate disposal of uranium compounds and possible nuclear leakage due to reactor disasters (Gongalsky 2003) become growing concern due to its high radioactivity, toxicity and bioavailability.(Aschner and Jiang 2009; Brugge et al. 2005; Domingo 2001b; Stojanovic et al. 2012). As its toxicity even in low concentrations can severely endanger human health, there is high demand for development of an effective method for sensitive uranium contamination monitoring. Current uranium detection relies mainly on instrumental methods, such as atomic emission spectrometry (AAS) (Jamali et al. 2006), inductively coupled plasma mass spectrometry (ICP-MS),(Wang et al. 2006) X-ray fluorescence (XRF) (Misra et al. 2010), fluorescence spectroscopy (Kushwaha et al. 2012; Sugitani et al. 1980; Yang et al. 2015) and surface-enhanced Raman spectroscopy (SERS) (Ruan et al. 2007). Although these methods offer excellent sensitivity, they require expensive equipment with high running cost and skilled operators.

DNAzymes are artificially evolved oligonucleotides which can be used as sensors for their specific targets (Gong et al. 2015; Lu 2002; Willner et al. 2008). Lu group reported the discovery of a DNAzyme for detection of uranyl $\left(\mathrm{UO}_{2}{ }^{2+}\right)$ with ultrahigh sensitivity and selectivity based on the cleavage of substrate strand by enzyme strand upon recognition of $\mathrm{UO}_{2}{ }^{2+}$ (Liu et al. 2007). The sensor was applied in the fluorescent (He and Lu 2011; Wu et al. 2013; Zhang et al. 2015b) and electrochemical detection (Ma et al. 2014; Peled et al. 2015) of $\mathrm{UO}_{2}{ }^{2+}$ after modification with 
fluorescent and electrochemical tags, respectively. Colorimetric methods were also developed with gold nanoparticles (AuNPs) (Lee et al. 2008). While the DNAzyme based sensors are cost-effective and readily fabricated, they are still not suitable for routine analysis in the field without sophisticated instruments. Therefore, it is desirable to develop a method to detect uranium in a speedy, inexpensive and portable way for field uranium contamination monitoring.

As a platform for portable detection, stimulus-responsive hydrogels utilize a synthetic polymer as scaffold and functional DNA as crosslinker (Guo et al. 2013; Lin et al. 2011; Yang et al. 2011; Yan et al. 2013; Zhu et al. 2010). The platform exhibits significant advantages for sensitive, seletive, cost-effective and portable detection (Yan et al. 2013; Zhu et al. 2010). In this work, we designed and synthesized a uranyl ion responsive hydrogel using a $\mathrm{UO}_{2}{ }^{2+}$ dependent DNAzyme and its substrate strand to crosslink DNA-grafted polyacrylamides for rapid, sensitive, and portable detection of $\mathrm{UO}_{2}{ }^{2+}$. The activation of DNAzyme by $\mathrm{UO}_{2}{ }^{2+}$ enables the cleavage of the substrate sequence, which subsequently destabilizes the hydrogel, which releases the initially encapsulated AuNPs for colorimetric detection. Lake water was used to verify that the $\mathrm{UO}_{2}{ }^{2+}$ responsive hydrogel is applicable in real water samples. The detection of $\mathrm{UO}_{2}{ }^{2+}$ in lake water was also carried out with a previously developed V-chip system (volumetric bar chart chip) (Huang et al. 2014; Zhu et al. 2014) by encapsulating Au-Pt nanoparticles (Au@AuPtNPs) in the hydrogel. The limit of detection reached $37 \mathrm{nM}$, which is lower than the EPA (United States Environmental Protection Agency) limit for uranium in drinking water, establishing a speedy, inexpensive, portable, and quantitative detection method for in-field detection of $\mathrm{UO}_{2}{ }^{2+}$. 


\section{Experimental}

\subsection{Materials and reagents}

All reagents for DNA synthesis (including A-TOM-CE phosphoramidite) were purchased from Glen Research (Sterling, VA, USA). Uranyl (VI) acetate was purchased from SPI supplies (Westchester, PA, USA). Acetate acid, 2-(N-morpholino) ethanesulfonic acid (MES), sodium chloride and acetonitrile were purchased from J\&K Scientific (Shanghai, China). Acrylamide, ammonium persulfate (APS), and tetramethylethylenediamine (TEMED) were purchased from Sigma-Aldrich (St. Louis, MO, USA). Other reagents were purchased from Sinopharm Chemical Reagents (Shanghai, China).

\subsection{Acrydite phosphoramidite synthesis}

Methacrylic acid (440 mg, $5 \mathrm{mmol}$ ), dicyclohexylcarbodiimide (DCC, $1356 \mathrm{mg}, 6 \mathrm{mmol}$ ), N-hydroxybenzotriazole (HOBT, $59.5 \mathrm{mg}, 0.5 \mathrm{mmol}$ ) and 6-amino-1-hexanol (585 mg, $5 \mathrm{mmol}$ ) were dissolved in $4 \mathrm{~mL} \mathrm{~N}, \mathrm{~N}$-dimethylformamide (DMF) and incubated overnight while stirring under an $\mathrm{N}_{2}$ atmosphere. After removing the precipitate of dicyclohexylurea (DCU) by filtration, water/acetic ether mixed liquor were used to remove residual DMF, and extraction of the product by acetic ether. The organic extract was dried over anhydrous magnesium sulfate and purified by column chromatography. The product $(213 \mathrm{mg}, 1.15 \mathrm{mmol})$ was dissolved in dichloromethane (DCM) and cooled to $0{ }^{\circ} \mathrm{C}$. After dropwise addition of $0.55 \mathrm{ml} \mathrm{N}, \mathrm{N}$-diisopropylethylamine (DIPEA) and $\quad 0.28 \quad \mathrm{ml}$ 2-cyanoethyl N,N-diisopropylchlorophosphoramidite $\left(\mathrm{P}-(\mathrm{N}-\mathrm{iPr})_{2}\left(\mathrm{OCH}_{2} \mathrm{CH}_{2} \mathrm{CN}\right)\right)$, the mixture was incubated at $0{ }^{\circ} \mathrm{C}$ in the dark for $4 \mathrm{hr}$. The product was obtained by column chromatography and dried to a colorless oil. 31P NMR (400 MHz, $\left.\mathrm{CDCl}_{3}\right): 147(\mathrm{~s}, 1 \mathrm{P})$. 


\subsection{Modified DNA synthesis}

All DNAs used in the work were synthesized on a Polygen GmbH 12-Column DNA Synthesizer. DNA sequences used for this work are shown in Fig. 1 and Table S1. Strands A1 and strand A2 were acrydite-modified DNAs. Common DNAs (except for acrydite DNAs) were deprotected by ammonia/methylamine ( $\mathrm{v} / \mathrm{v}=1 / 1)$ solution at $65{ }^{\circ} \mathrm{C}$ for $20 \mathrm{~min}$ after synthesis. They were purified by high-performance liquid chromatography (HPLC) using a reversed-phase C18 column. Two solutions with varying polarities were used for gradient elution: $0.1 \mathrm{M}$ triethylamine acetate ( $\mathrm{pH}$ 7.0) and HPLC-grade acetonitrile. Due to the lack of a hydrophobic group (4,4'-dimethoxytriphenylmethyl group) in acrydite-modified DNA, the elution gradient differed. Non-modified DNAs were eluted with a gradient of acetonitrile from 10 to 65 percent with times from 4 min to $35 \mathrm{~min}$. Acrydite-modified DNAs were eluted in a gradient of acetonitrile varying from 12 to 27 percent with the time from $4 \mathrm{~min}$ to $55 \mathrm{~min}$. After vacuum removal of the solvent, the primitive non-modified DNA was detritylized by $80 \%(\mathrm{v} / \mathrm{v})$ acetate acid. Acrydite-modified DNA did not require detritylization, and was desalted 3 times with a 3k NMWL (nominal molecular weight limit) Millipore ultracentrifugal filter. Substrate strand modified in the middle with A-TOM-phosphoramidite was purified as follows. The abovementioned steps before detritylation were performed the same as non-modified DNA. After that, the protecting 2'-O-triisopropylsilyloxymethyl (TOM) group was removed by addition of $125 \mu \mathrm{L}$ triethylamine trihydrofluoride in $100 \mu \mathrm{L}$ DMSO for $3 \mathrm{hr}$ at $65{ }^{\circ} \mathrm{C}$. Then, $25 \mu \mathrm{L} 3 \mathrm{M}$ sodium acetate and $1 \mathrm{~mL}$ butyl alcohol were used to precipitate DNA at $-20{ }^{\circ} \mathrm{C}$ after 30 min incubation. All product DNA was desalted 3 times by a 3k NMWL (nominal molecular weight limit) Millipore ultracentrifugal filter and dissolved by RNase free water. The concentration was quantified by the absorbance at 
$260 \mathrm{~nm}$, and the stock solution was stored at $-20{ }^{\circ} \mathrm{C}$ for future use.

\subsection{Preparation of DNA-grafted polyacrylamide chains}

Stock solutions of strand A1 and A2 grafted polyacrylamide chains were prepared in final concentration of $0.5 \mathrm{mM}$ separately in PCR tubes. The tubes contained $4 \%$ acrylamide in total volume of $40 \mu \mathrm{L}$. After degassing by air pump for $10 \mathrm{~min}, 0.56 \mu \mathrm{L}$ freshly prepared $10 \%(\mathrm{~m} / \mathrm{v})$ APS and $1.12 \mu \mathrm{L}$ TEMED (5\%, v/v) solution were added to the abovementioned tubes. Immediately after the mixing of APS and TEMED, the tubes were vacuumed by an air pump in a vacuum desiccator for another $15 \mathrm{~min}$ at $37{ }^{\circ} \mathrm{C}$ to copolymerize the acrydite DNA and acrylamide, yielding DNA-grafted chains pA1 and pA2. Ultracentrifugal filtration with $100 \mathrm{k}$ NMWL removed the unpolymerized strands.

\subsection{Modification of nanoparticles}

The synthesis of nanoparticles is described in the supporting information. The unprotected nanoparticles are not suitable for encapsulation in the hydrogel due to the high concentration of salt, which would induce nanoparticle aggregation. Therefore, AuNPs were protected by thiol-polyethylene glycol (PEG-SH, MW 5000). Briefly, $2.5 \mathrm{nM}$ AuNPs were incubated with 1 $\mu \mathrm{M}$ PEG-SH at $37{ }^{\circ} \mathrm{C}$ for $30 \mathrm{~min}$. After centrifuging and redispersing 3 times, RNase-free water was used to dilute AuNPs to $150 \mathrm{nM}$. Au@AuPtNPs were neutralized in $10 \mathrm{mM}$ phosphate buffer first. After the addition of $0.01 \%$ Tween 20, $1 \mu \mathrm{M}$ PEG-SH and $2 \mu \mathrm{M}$ T15-SH (3' thiol polyThymine) were added and vortexed vigorously. Finally, $100 \mathrm{mM} \mathrm{MgCl}_{2}$ were used to age $\mathrm{Au} @ \mathrm{AuPtNPs}$ for $30 \mathrm{~min}$ at $37{ }^{\circ} \mathrm{C}$. The same centrifuging and redispersing steps were implemented and the final stock concentration of Au@AuPtNP was 100 nM.

\subsection{Preparation of $\mathrm{UO}_{2}{ }^{2+}$ responsive hydrogel.}

To prepare the hydrogel, pA1: pA2: substrate strand: enzyme strand were mixed in a molar ratio of $100 \mu \mathrm{M}$ : $100 \mu \mathrm{M}$ : $50 \mu \mathrm{M}$ : $100 \mu \mathrm{M}$. The $30 \mathrm{nM}$ AuNPs or $10 \mathrm{nM} \mathrm{Au} @$ AuPtNPs were encapsulated in the formed hydrogel. The mixture was alternately vortexed vigorously and incubated at $65{ }^{\circ} \mathrm{C}$ for $1 \mathrm{~min}$ three times to generate a homogenous hydrogel. Finally, the hydrogel 
was slowly cooled to room temperature. The total volume of hydrogel was $10 \mu \mathrm{L}$. The reaction buffer was $50 \mathrm{mM}$ MES buffer (pH 5.5, containing $250 \mathrm{mM} \mathrm{NaCl}$ ).

\subsection{Detection of $\mathrm{UO}_{2}{ }^{2+}$ by colorimetric essay}

The AuNP-encapsulated hydrogel was formed in the previous step. Fifty $\mu \mathrm{LUO}_{2}{ }^{2+}$ samples in different concentrations were added to the hydrogel and allowed to incubate at $25{ }^{\circ} \mathrm{C}$ while shaking at $150 \mathrm{rpm}$ for $2 \mathrm{hr}$. Color changes were observed in the supernatant of the hydrogel indicating the $\mathrm{UO}_{2}{ }^{2+}$ induced collapse of the hydrogel and release of the internal AuNPs. Supernatant absorbance at $520 \mathrm{~nm}$ of AuNPs showed the concentration-dependent collapse of hydrogel by $\mathrm{UO}_{2}{ }^{2+}$.

\subsection{V-chip visual detection of $\mathrm{UO}_{2}{ }^{2+}$}

Fifty $\mu \mathrm{L}$ volumes of different concentrations of $\mathrm{UO}_{2}{ }^{2+}$ were loaded onto $10 \mu \mathrm{L}$ Au@AuPtNP-encapsulated hydrogel. The reaction between $\mathrm{UO}_{2}{ }^{2+}$ and the hydrogel was allowed to proceed for $2 \mathrm{hr}$ at $25{ }^{\circ} \mathrm{C}$ while shaking at $150 \mathrm{rpm}$. The resultant supernatant with released Au@AuPtNPs was then transferred to the sample position on the V-Chip. After sliding the top layer of the chip, Au@AuPtNPs in the sample were mixed with $\mathrm{H}_{2} \mathrm{O}_{2}$ to produce $\mathrm{O}_{2}$. A large volume of $\mathrm{O}_{2}$ was generated to push forward the ink bar through the connected vertical channel. The length of the ink bar was used to calculate the concentration of $\mathrm{UO}_{2}{ }^{2+}$.

\section{Results and Discussion}

\subsection{Working principle of uranyl ion responsive hydrogel}

The working principle of the uranyl ion responsive hydrogel is schematically shown in Fig. $\mathbf{1}$

The enzyme strand and substrate strand form a stem-loop complex which is designed to hybridize with two types of DNA polyacrylamide chains to form the DNAzyme hydrogel. AuNPs are encapsulated in the hydrogel during the formation process to indicate the integrity of hydrogel. The hydrogel stays stable until uranyl ion is present in the sample. After activation of the enzyme by uranyl ion, the enzyme strand starts to cleave the substrate strand, inducing the dissociation of the stem-loop structure. Thus the crosslinking ratio of the hydrogel is gradually decreased, and 
subsequently the hydrogel is destabilized to release the encapsulated AuNPs. Meanwhile, the uranyl ion binding to the original enzyme and substrate complex will dissociate to bind to another preformed enzyme and substrate complex to facilitate another cycle of cleaving reaction. As a result, a small amount of uranyl ion induces the release of large amounts of AuNPs, which turn the supernatant from colorless to red. Therefore, the concentration of uranyl ion is indicated by the supernatant color change. The AuNPs encapsulated in the hydrogel can also be replaced by enzymes or PtNPs to catalyze the generation of large amounts of signaling molecules for a variety of readout devices, such as a gluocometer, distance-based microfluidic chip etc. (Yan et al. 2013;

Zhu et al. 2014)

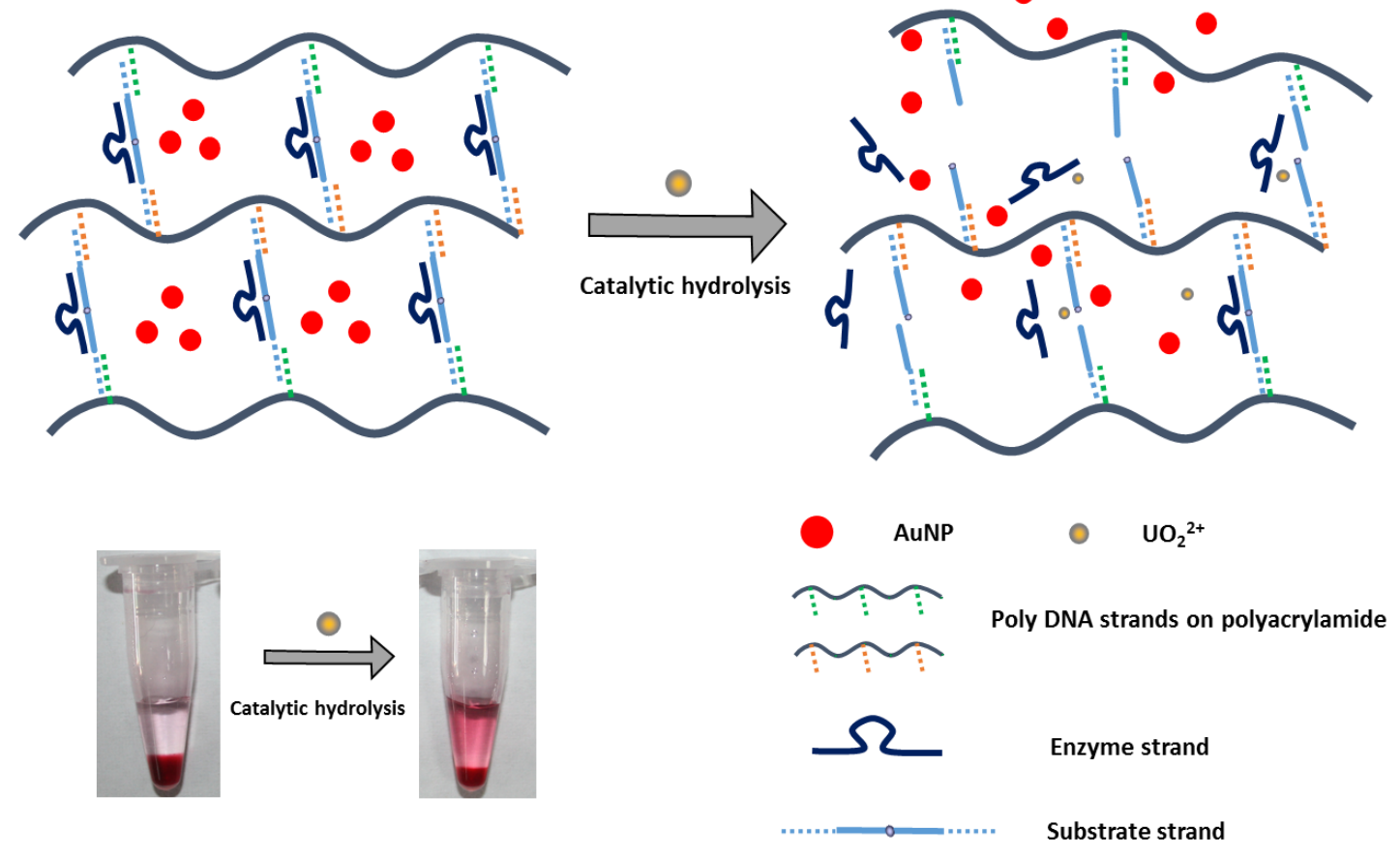

Fig. 1 Working principle of the uranyl ion responsive hydrogel. AuNPs encapsulated in hydrogel are released in the presence of uranyl ion due to the cleavage of the crosslinking substrate strands. The subsequent result can be monitored by naked eye.

\subsection{Sequence design and optimization of the hydrogel}

The structure of the enzyme and substrate strand complex and construction of hydrogel are 
shown in Fig. 2. The hydrogel is composed of four strands of DNAs, including polymerized A1 (pA1), polymerized A2 (pA2), enzyme strand (Enz) and substrate strand (Sub). Sequence A1, enzyme strand, and A2 are designed to hybridize to the adjacent sequences on the substrate. To enable a stable hydrogel construction, A1 and A2 were set to 14 nucleotides each to ensure stable hybridization with the full substrate sequence to crosslink pA1 and pA2 for formation of a stable hydrogel. As shown in Fig. S1C, the melting temperature $\left(\mathrm{T}_{\mathrm{m}}\right)$ of Enz/Sub is about $50{ }^{\circ} \mathrm{C}$, thus stable formation of Enz/Sub at room temperature is expected. On the other hand, once the substrate is cleaved by activated enzyme, either of the resulting daughter sequences can dissociate from the enzyme sequences to ensure rapid dissolution of the hydrogel. Unfortunately, the $\mathrm{T}_{\mathrm{m}} \mathrm{s}$ of full length Enz with two daughter sequences of Sub (Sub1 and Sub2) after the cleavage are $40{ }^{\circ} \mathrm{C}$ and $45^{\circ} \mathrm{C}$, respectively (Fig. S1C). At this binding strength, it is expected that the DNAzyme can still bind to Sub1 and Sub2 at room temperature, leaving the hydrogel undissolved after target- induced enzymatic cleavage. Therefore, the enzyme sequence was shortened by 1, 2, 3 nucleotides respectively at the 3' end to obtain Enz-1, Enz-2, Enz-3, as shown in Table S1. As a result, the $T_{m} \mathrm{~s}$ of sub1 with Enz-1, Enz-2, and Enz-3 were significantly decreased to less than $20{ }^{\circ} \mathrm{C}$ with Enz-3 having the smallest binding ability to sub1 (Fig. S1A). More importantly, all three shortened enzyme sequences retain their activity to cleave the substrate sequence in the presence of $\mathrm{UO}_{2}{ }^{2+}$ (Fig. S1D). As a result, Enz-3 was chosen as the enzyme sequence for hydrogel synthesis.

A
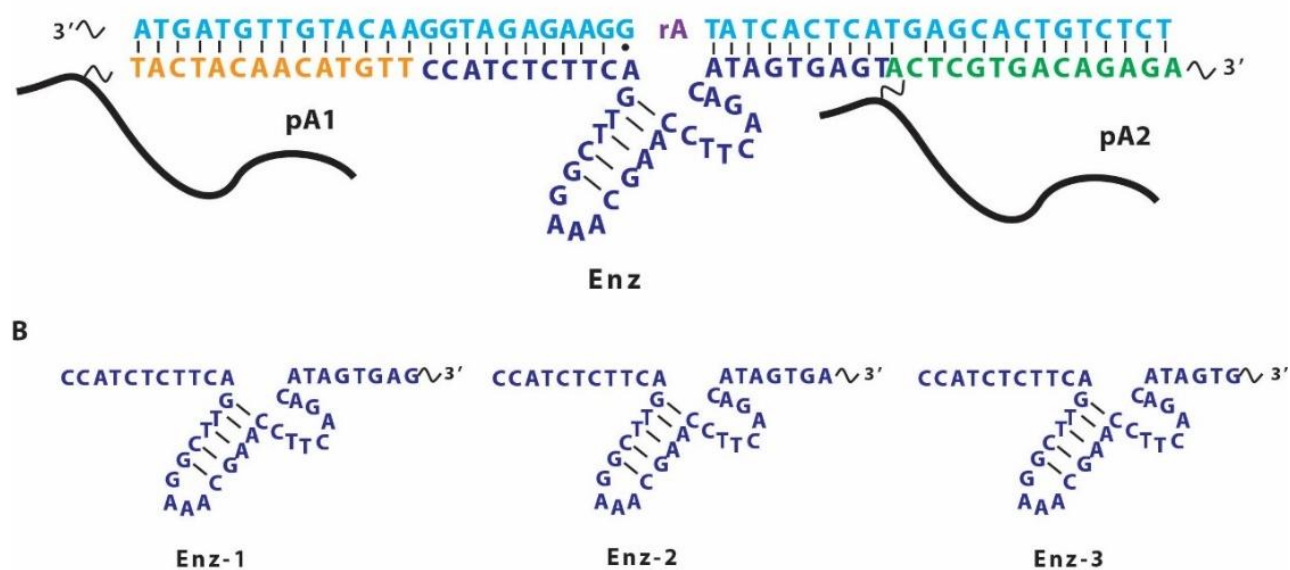

Fig. 2. Design of hydrogel-forming DNA and optimized sequences for the $\mathrm{UO}_{2}{ }^{2+}$ responsive hydrogel. (A) The hydrogel is composed of 4 strands of DNA: pA1, pA2, enzyme strand (Enz) and substrate strand (Sub). (B) The Enz was cut down from original sequence by 1, 2, 3 nucleotides at 3' end of strands to gain Enz-1, Enz-2, Enz-3. 


\subsection{Sensitive and Selective Colorimetric Detection of $\mathrm{UO}_{2}{ }^{2+}$}

Four types of DNA were added together in the tube to prepare $\mathrm{UO}_{2}{ }^{2+}$ responsive hydrogel. As shown in Fig. S2, gold nanoparticles were stably trapped inside the hydrogel, suggesting the successful preparation of stable hydrogel. To test the target sensing behavior of the hydrogel, AuNP-encapsulated hydrogel was reacted with $1 \mu \mathrm{M} \mathrm{UO}_{2}{ }^{2+}$. The result showed that in absence of target, no leakage of AuNPs into the supernatant was observed, while steady increase of AuNP absorption from the supernatant was recorded upon addition of target. This experiment clearly proved that the hydrogel was responsive to $\mathrm{UO}_{2}{ }^{2+}$. Afterwards, the hydrogel was treated with various concentrations of $\mathrm{UO}_{2}{ }^{2+}$ to monitor the target-responsive performance. As shown in Fig. 3A, with increasing concentrations of $\mathrm{UO}_{2}{ }^{2+}$, the volume of hydrogel became smaller, and the color of the supernatant became darker, indicating that the recognition of $\mathrm{UO}_{2}{ }^{2+}$ triggered the cleavage reaction and thus the disruption of the hydrogel. Even with $100 \mathrm{nM} \mathrm{UO}_{2}{ }^{2+}$, a clear color change can be observed, which is lower than the requirement of EPA (United States Environmental Protection Agency, 130 nM). Further UV-Vis spectroscopy measurement results shown in Fig. 3B offer more precise quantitative results. The target-responsive hydrogel can detect $\mathrm{UO}_{2}{ }^{2+}$ from 0 $800 \mathrm{nM}$. The linear range is between $0-200 \mathrm{nM}$ with the limit of detection (LOD) of $14 \mathrm{nM}$ based on the $3 \sigma$ rule. 

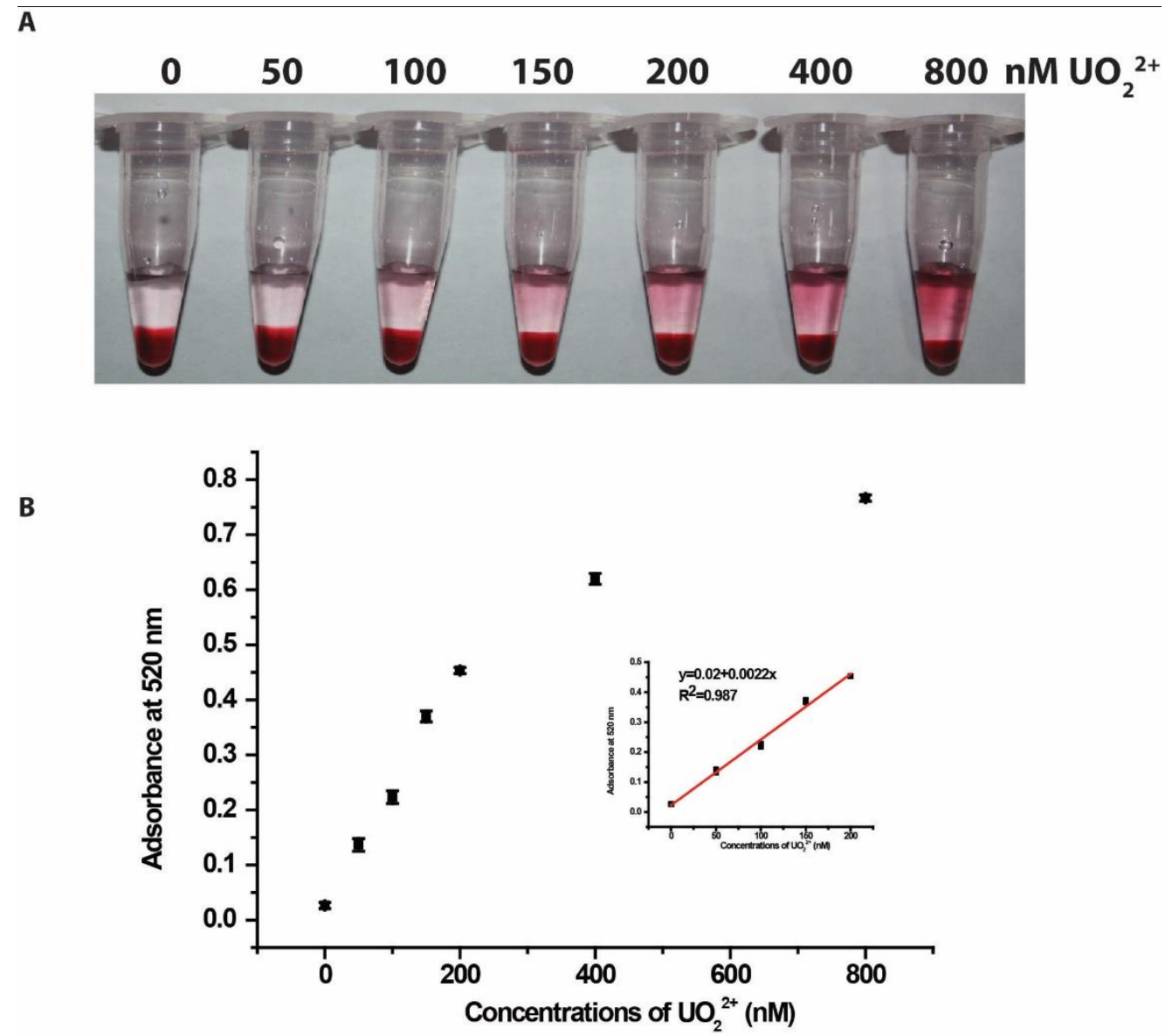

B

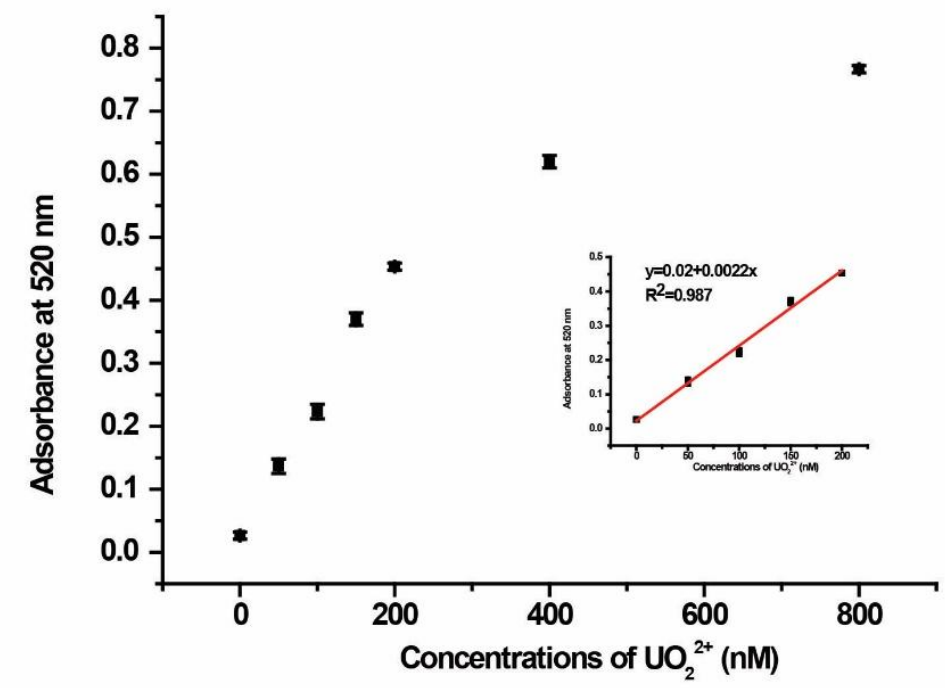

Fig. 3 (A) Sensitivity of hydrogel for $\mathrm{UO}_{2}{ }^{2+}$ detection. $\mathrm{UO}_{2}{ }^{2+}$ in the samples induces the decomposition of hydrogel to release the encapsulated AuNPs. The results can be observed by the naked eye. (B) Absorbance at $520 \mathrm{~nm}$ of supernatant corresponding to A. The linear range is observed between 0 and $200 \mathrm{nM}$.

Taking advantage of the high selectivity of the $\mathrm{UO}_{2}{ }^{2+}$ DNAzyme, our $\mathrm{UO}_{2}{ }^{2+}$ responsive hydrogel displayed good selectivity against other cations. As shown in Fig. 4A, in the presence of other common cations with even 100-fold higher concentration than that of $\mathrm{UO}_{2}{ }^{2+}$, the uranyl ion responsive hydrogel did not show any color change in the supernatant, proving that the presence of other cations does not contribute to the output signal. Corresponding UV-Vis data in Fig. 4B clearly demonstrated that uranyl ion hydrogel did not react with cations other than $\mathrm{UO}_{2}{ }^{2+}$. The results confirmed the fidelity of the uranyl ion hydrogel in seletive detection of $\mathrm{UO}_{2}{ }^{2+}$. 
A

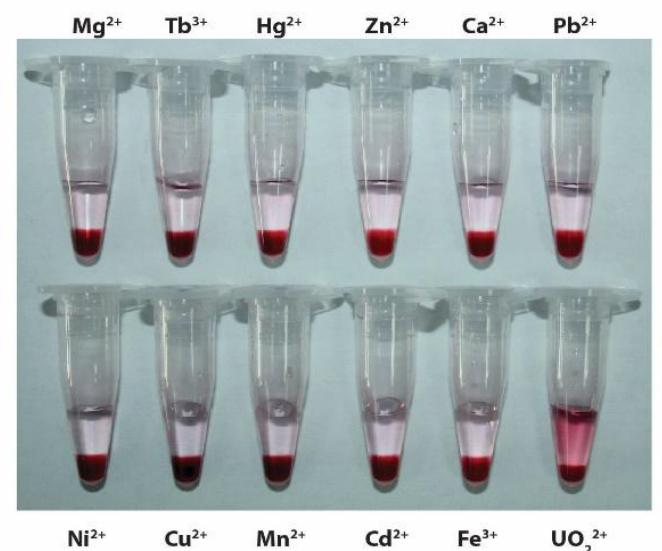

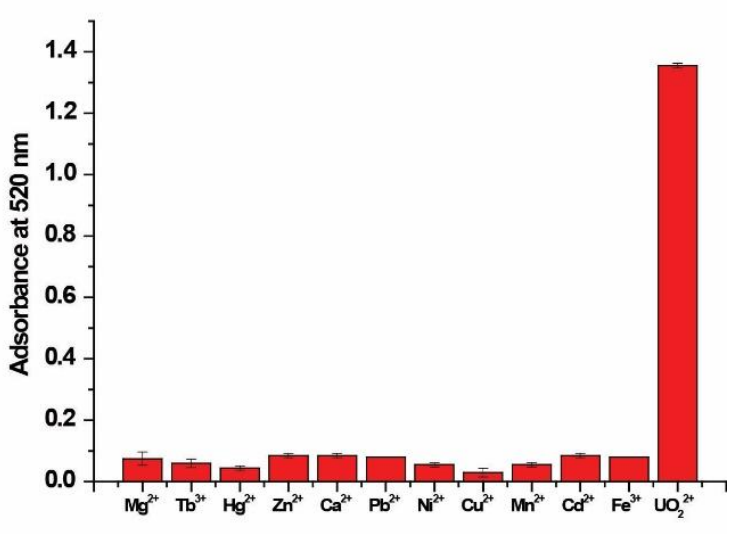

Fig. 4 Selectivity of uranyl ion hydrogel. (A) Response of hydrogel to $100 \mu \mathrm{M}$ cations and $1 \mu \mathrm{M}$ $\mathrm{UO}_{2}{ }^{2+}$. (B) The corresponding supernatant absorbance at $520 \mathrm{~nm}$.

\subsection{Trace $\mathrm{UO}_{2}{ }^{2+}$ detection in lake samples with the hydrogel.}

Natural water can be contaminated with uranium due to poorly controlled mining and processing or leakage from nuclear power plants. The uranyl ion responsive hydrogel was applied to detect trace $\mathrm{UO}_{2}{ }^{2+}$ in natural lake water which was used as a complex matrix. Lake water not polluted by uranium was sampled from Furong Lake on Xiamen University campus by simply filtering with $0.25 \mu \mathrm{m}$ filter. Different concentrations of $\mathrm{UO}_{2}{ }^{2+}$ were spiked into the lake sample. As shown in Fig. 5, similar to the results with buffer only as the matrix, the supernatant colors gradually turned deeper as the concentration of $\mathrm{UO}_{2}{ }^{2+}$ increased. UV-Vis measurements of the supernatant solution showed that the linear range for $\mathrm{UO}_{2}{ }^{2+}$ is between 0 to $300 \mathrm{nM}$, with LOD of $20 \mathrm{nM}$. 
A

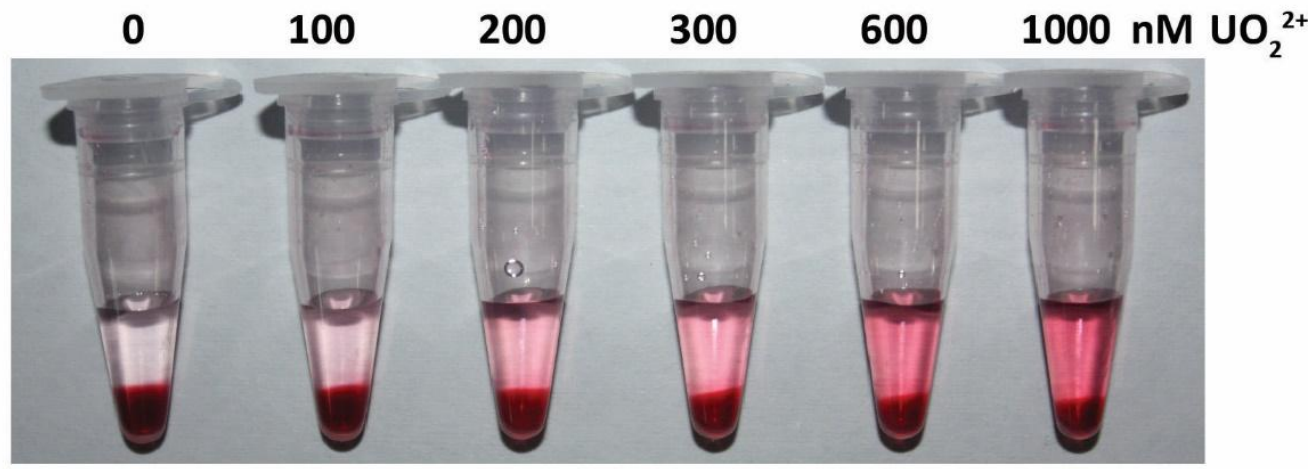

B

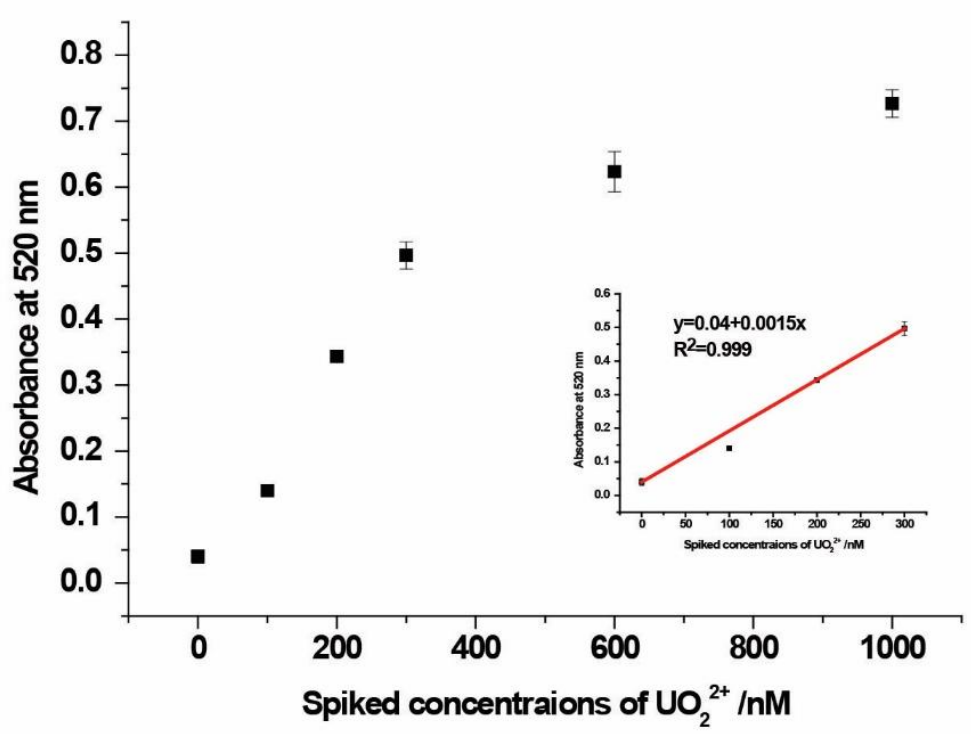

Fig. 5 Sensitive detection of $\mathrm{UO}_{2}{ }^{2+}$ in spiked lake water. (A) Different concentrations of $\mathrm{UO}_{2}{ }^{2+}$ were spiked in natural lake water to react with the AuNP encapsulated hydrogel. (B) The corresponding supernatant absorbance of AuNPs at $520 \mathrm{~nm}$.

\subsection{Hydrogel coupled with V-Chip for Quantitative Detection of $\mathrm{UO}_{2}{ }^{2+}$}

Colorimetric detection was limited to semi-quantitation due to the color intensity limitations of the naked eye. Translating the target recognition to visual length signal is a good alternative for a portable quantitative method. The lengths are in accordance with target concentration due to the specificity of the reaction achieved by molecular recognition. The V-Chip (volumetric bar chart 
chip) has been proven to be a good visual distance-based detection apparatus for sensitive detection of cocaine, lead ion, etc. (Huang et al. 2014; Zhu et al. 2014). The working principle of V-Chip has been described previously (Huang et al. 2014; Zhu et al. 2014). Briefly, as shown in Fig. S3, the V-Chip is composed of two glass slides, top layer and bottom layer separately. Each slide is patterned with different disconnected channels. The patterns are designed so that when assembling the two glasses, the disconnected channels are connected into a thoroughly horizontal or vertical connected channel. The choices are controlled by the relative positions between two glass slides.

We further exploited this device to realize quantitative detection of $\mathrm{UO}_{2}{ }^{2+}$ by ink bar length. When the horizontal direction was connected, the chip was loaded with sample, $\mathrm{H}_{2} \mathrm{O}_{2}$ and dye in different chambers located at different positions of the chip. Samples were obtained from the hydrogel supernatant following the reaction of the Au@AuPtNP-encapsulated hydrogel with different concentrations of $\mathrm{UO}_{2}{ }^{2+}$. Immediately after sliding of the top layer, the sample chamber of the top layer was brought to the $\mathrm{H}_{2} \mathrm{O}_{2}$ chamber of the bottom layer to trigger the enzymatic decomposition of $\mathrm{H}_{2} \mathrm{O}_{2}$ to generate $\mathrm{O}_{2}$ by $\mathrm{Au} @$ AuPtNPs. Meanwhile the sliding changed the horizontal connecting channels into vertical connecting channels and thus the resultant $\mathrm{O}_{2}$ pushed the ink bar via the vertical connecting channels. By reading the increase in ink bar length, the $\mathrm{UO}_{2}{ }^{2+}$ concentration was visually determined. The concentrations of $\mathrm{UO}_{2}{ }^{2+}$ spiked in lake water were measured in V-chip as shown in Fig. 6. The $\mathrm{UO}_{2}{ }^{2+}$ concentration was linearly correlated to the ink bar length in the range of $0-600 \mathrm{nM}$. The detection limit of $37 \mathrm{nM}$ based to the $3 \sigma$ rule was comparable to that of the previously reported colorimetric method.(Lee et al. 2008; Zhang et al. 2015a) The results of V chip detection were also verified by the traditional ICP-MS method to 
confirm the accuracy. The agreement between V chip results and ICP-MS results proved the utility of our portable $\mathrm{V}$ chip method for accurate and sensitive detection of $\mathrm{UO}_{2}{ }^{2+}$ (Table S2).

A

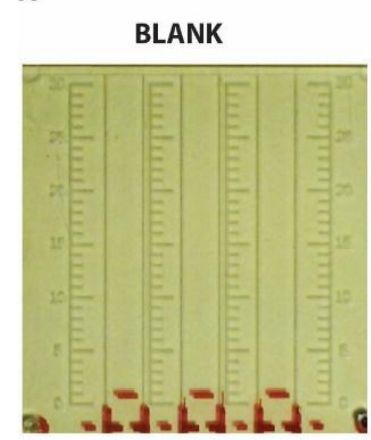

$300 \mathrm{nM}$

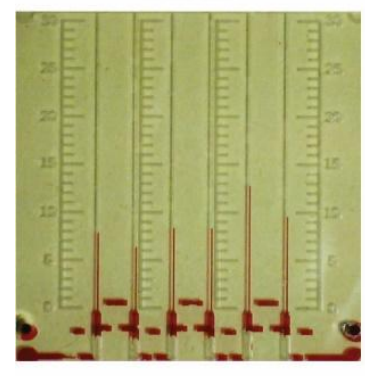

$50 \mathrm{nM}$

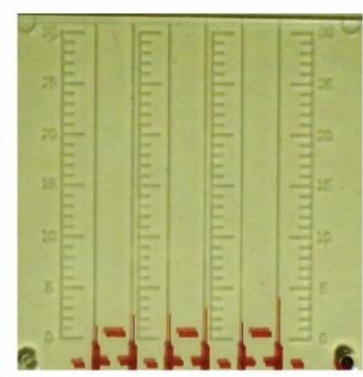

$600 \mathrm{nM}$

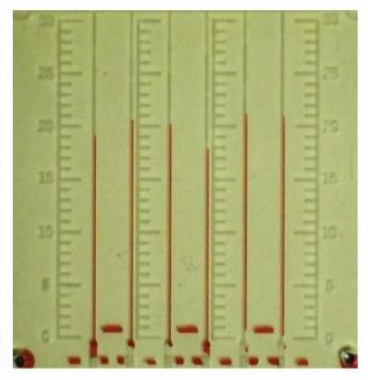

$100 \mathrm{nM}$

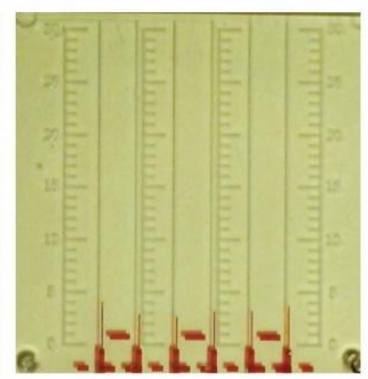

B

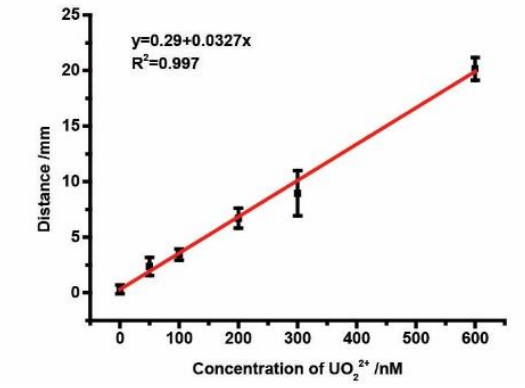

Fig. 6. Sensitive and portable detection of $\mathrm{UO}_{2}{ }^{2+}$ on a V-Chip. (A) Different concentrations of $\mathrm{UO}_{2}{ }^{2+}$ spiked in lake water induce different lengths of the ink bars. (B) The linear range by V-chip was $0-600 \mathrm{nM}$ with the LOD of $37 \mathrm{nM}$.

\section{Conclusions}

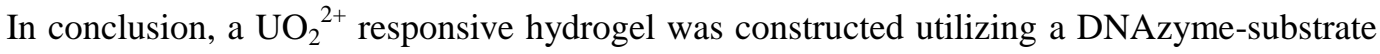
complex structure as crosslinker to hybridize with DNA-grafted polyacrylamide chains. The presence of $\mathrm{UO}_{2}{ }^{2+}$ activates the DNAzyme's activity to cleave the substrate strand, and the breakage of the substrate strand and dissociation of the enzyme strand lead to the collapse of hydrogel. The hydrogel encapsulating AuNPs allows colorimetric detection of $\mathrm{UO}_{2}{ }^{2+}$ concentrations as low as $100 \mathrm{nM}$ by naked eye portably and selectively. To overcome the lack of accuracy by colorimetric essay, a distance-based V-chip detecting apparatus was incorporated with the hydrogel sensing system for portable and quantitative detection of $\mathrm{UO}_{2}{ }^{2+}$ in lake water sample with a detection limit of $37 \mathrm{nM}$, which is lower than the EPA limit (130 nM). This result 
was consistent with that by the traditional ICP-MS method. The target responsive hydrogel incorporating V-chip system offers a promising platform to detect $\mathrm{UO}_{2}{ }^{2+}$ in a sensitive, specific, portable, user-friendly, and quantitative manner for monitoring uranium contamination in field work.

\section{Acknowledgments}

We thank the National Science Foundation of China (21325522, 21205100, 21275122), National Found for Fostering Talents of Basic Science (NFFTBS, J1310024), and National Instrumentation Program (2011YQ03012412), for their financial support.

\section{References}

Aschner, M., Jiang, G.C.T., 2009. J. Toxical. Env Heal. B. 12(7), 525-539.

Brugge, D., de Lemos, J.L., Oldmixon, B., 2005. Rev. Environ. Health 20(3), 177-193.

Domingo, J.L., 2001a. Reprod Toxicol 15(6), 603-609.

Domingo, J.L., 2001b. Reprod. Toxical. 15(6), 603-609.

Gong, L., Zhao, Z.L., Lv, Y.F., Huan, S.Y., Fu, T., Zhang, X.B., Shen, G.L., Yu, R.Q., 2015. Chem. Commun. 51(6), 979-995.

Gongalsky, K.B., 2003. Environ. Monit. Assess. 89(2), 197-219.

Guo, W., Orbach, R., Mironi-Harpaz, I., Seliktar, D., Willner, I., 2013. Small 9(22), 3748-3752.

He, Y., Lu, Y., 2011. Chem. Eur. J. 17(49), 13732-13742.

Huang, Y., Ma, Y., Chen, Y., Wu, X., Fang, L., Zhu, Z., Yang, C.J., 2014. Anal. Chem. 86(22), 11434-11439.

Jamali, M.R., Assadi, Y., Shemirani, F., Hosseini, M.R.M., Kozani, R.R., Masteri-Farahani, M., Salavati-Niasari, M., 2006. Anal. Chim. Acta 579(1), 68-73.

Kushwaha, S., Rao, S.A., Sudhakar, P.P., 2012. Inorg. Chem. 51(1), 267-273.

Lee, J.H., Wang, Z.D., Liu, J.W., Lu, Y., 2008. J. Am. Chem. Soc. 130(43), 14217-14226.

Lin, H., Zou, Y., Huang, Y., Chen, J., Zhang, W.Y., Zhuang, Z., Jenkins, G., Yang, C.J., 2011. Chem. Commun. 47(33), 9312-9314.

Liu, J.W., Brown, A.K., Meng, X.L., Cropek, D.M., Istok, J.D., Watson, D.B., Lu, Y., 2007. P. Natl Acad. Sci. USA 104(7), 2056-2061.

Lu, Y., 2002. Chem. Eur. J. 8(20), 4589-4596.

Ma, D.D., Yuan, Y.L., Xiao, X.L., Gao, Y.Y., Li, Y.H., Xu, W.H., Long, W., 2014. J. Radioanal. Nucl. Chem. 299(3), 1911-1919.

Misra, N.L., Dhara, S., Ovari, M., Zaray, G., Aggarwal, S.K., Varga, I., 2010. Spectrochim. Acta. B 65(6), 457-460.

Peled, Y., Krent, E., Tal, N., Tobias, H., Mandler, D., 2015. Anal. Chem. 87(1), 768-776. 
Ruan, C.M., Luo, W.S., Wang, W., Gu, B.H., 2007. Anal. Chim. Acta 605(1), 80-86.

Stojanovic, M., Stevanovic, D., Milojkovic, J., Mihajlovic, M.L., Lopicic, Z., Sostaric, T., 2012. Water Air Soil Pollut. 223(1), 135-144.

Sugitani, Y., Nomura, H., Nagashima, K., 1980. Bull. Chem. Soc. Jpn. 53(9), 2677-2678.

Wang, L., Yang, Z.M., Gao, J.H., Xu, K.M., Gu, H.W., Zhang, B., Zhang, X.X., Xu, B., 2006. J. Am. Chem. Soc. 128(41), 13358-13359.

Willner, I., Shlyahovsky, B., Zayats, M., Willner, B., 2008. Chem. Soc. Rev. 37(6), 1153-1165.

Wu, P.W., Hwang, K.V., Lan, T., Lu, Y., 2013. J. Am. Chem. Soc. 135(14), 5254-5257.

Yan, L., Zhu, Z., Zou, Y., Huang, Y., Liu, D., Jia, S., Xu, D., Wu, M., Zhou, Y., Zhou, S., Yang, C.J., 2013. J. Am. Chem. Soc. 135(10), 3748-3751.

Yang, C.-T., Han, J., Gu, M., Liu, J., Li, Y., Huang, Z., Yu, H.-Z., Hu, S., Wang, X., 2015. Chem. Commun. 51(59), 11769-11772.

Yang, L., Zhang, X., Ye, M., Jiang, J., Yang, R., Fu, T., Chen, Y., Wang, K., Liu, C., Tan, W., 2011. Adv. Drug Deliver. Rev. 63(14-15), 1361-1370.

Zhang, D.Y., Chen, Z., Omar, H., Deng, L., Khashab, N.M., 2015a. Acs Appl Mater Inter 7(8), 4589-4594.

Zhang, H.Y., Ruan, Y.J., Lin, L., Lin, M.G., Zeng, X.X., Xi, Z.M., Fu, F.F., 2015b. Spectrochim. Acta. A 146, 1-6.

Zhu, Z., Guan, Z., Jia, S., Lei, Z., Lin, S., Zhang, H., Ma, Y., Tian, Z.-Q., Yang, C.J., 2014. Angew. Chem. Int. Edit. 53(46), 12503-12507.

Zhu, Z., Wu, C., Liu, H., Zou, Y., Zhang, X., Kang, H., Yang, C.J., Tan, W., 2010. Angew. Chem. Int. Edit. 49(6), 1052-1056. 